\title{
Creatinine Clearance Adjusted for BSA
}

National Cancer Institute

\section{Source}

National Cancer Institute. Creatinine Clearance Adjusted for BSA. NCI Thesaurus. Code C147324.

The determination of the clearance of creatinine and whose result is adjusted for body surface area. 